\title{
Non-optimality of the Myopic Decision Rule: The Case of a Two-Sector Open Economy*
}

\author{
TAPAN Biswas \\ Department of Economics, University of California, Santa Barbara, California 93106 \\ Received November 6, 1972 \\ This paper is concerned with optimal allocation of investment in a two-sector \\ open economy with non-shiftable capital. We have assumed a stationary popula- \\ tion and fixed terms of trade. It has been shown that, if the economy starts \\ with a small amount of capital in each sector, the myopic decision rule under \\ static expectations will not be compatible with the conditions of optimal in- \\ vestment allocation.
}

\section{INTRODUCTION}

A few years ago Arrow [1] observed that investment decisions made according to a myopic decision rule under perfect foresight result in an optimal capital policy provided investment is reversible. In case of irreversible investment the non-negativity constraint on gross investment may be effective somewhere on the optimal path. When this situation occurs, the equivalence of the myopic decision rule and the optimal capital policy breaks down. The treatment of the non-negativity constraint on gross investment was discussed in further detail by Arrow [2]. If we consider the optimal investment policy of an economy endowed with heterogeneous (or non-shiftable) capital, the non-negativity constraints on gross investments in different types of capital may be effective somem where on the optimal path. In such a case the myopic decision rule is not necessarily consistent with the optimal capital policy. Shell and Stiglitz [6] considered an economy producing only one type of good with the aid of two types of capital and labor. The production function was assumed to be of the Cobb-Douglas type. Because of this special assumption in

* This is a revised version of a chapter in my Ph.D. dissertation submitted to the University of Rochester in June, 1972. I am indebted to Professors Lionel W. McKenzie, Ronald W. Jones, Emmanuel Drandakis, and J. H. B. Kemperman for helpful com* ments. I would also like to thank J. Marshall and the referee of this journal for many valuable suggestions.

Originally publlished in Journal of Economic Theory (1974), 7, 115-121 
their model the critical path of capital accumulation (along which the rates of return on both types of capital are equal) is a straight line with positive slope. Once this critical path is reached, it is possible for the economy to stay on this path indefinitely. Since the critical path has this interesting property it follows from their discussion that investment decisions made under the myopic decision rule (with static expectations regarding the prices of capital goods) result in an optimal path of capital accumulation.

In this paper we shall consider a two-sector open economy where capital goods are not shiftable from one sector to the other. The terms of trade with the rest of the world are assumed to be fixed. Here the critical path (Rybczynski Locus), unlike the one considered by Shell-Stiglitz, has a negative slope. Hence, in this case it is not possible for the economy to stay on the critical path. It is shown below that in this case the myopic decision rule under static price expectations does not necessarily imply the optimal capital policy.

\section{The Problem}

Consider an economy which consists of a consumption goods sector $(F)$ and a manufacturing sector $(M)$. The production functions are denoted as follows:

$$
\begin{gathered}
F=F_{f}\left(K_{f}, L_{f}\right), \\
M=F_{m}\left(K_{m}, L_{m}\right),
\end{gathered}
$$

where $K_{j}$ and $L_{j}$ are the amounts of capital and labor used in the $j$-th sector. The production functions are assumed to be strictly concave with respect to their arguments. Capital cannot be shifted from one sector to the other but labor is a perfectly mobile factor of production. Production functions are subject to constant returns to scale and the total supply of labor is fixed. Hence, without any loss of generality we can write,

$$
L_{f}+L_{m}=1 \text {. }
$$

The economy is engaged in trade with a fixed terms of trade $P\left(=P_{f} / P_{m}\right)$. We assume that $P$ has a value such that both the commodities will be produced for some value of total capital stock $\left(K_{f}+K_{m}\right)$, if capital were shiftable from one sector to the other. Let $x_{f}$ and $x_{m}$ denote the amounts of the consumption goods and the manufacturing goods exported from the country. From the condition of balanced trade,

$$
P \cdot x_{f}+x_{m}=0 .
$$

Originally publlished in Journal of Economic Theory (1974), 7, 115-121 
Let $C$ and $I$ denote the amounts of consumption and investment respectively. Hence, by definition,

$$
\begin{aligned}
& C=F-x_{f}, \\
& I=M+P \cdot x_{f},
\end{aligned}
$$

We assume that the planners' objective is

$$
\operatorname{maximize} \int_{0}^{\infty} U(C) \cdot d t
$$

where $U^{\prime}(C)>0, U^{\prime \prime}(C)<0$. Also $B-U(C)>0$ for some $B>0$ and $0<C<\infty$. Following Pontryagin's maximum principle [5], we can write the Hamiltonian as:

$$
H=U(C)+\left[\Psi_{1} \lambda+\Psi_{2}(1-\lambda)\right]\left(M+P \cdot x_{f}\right),
$$

where $\dot{K}_{f}=\lambda l, \dot{K}_{m}=(1-\lambda) I$, and $\lambda \in[0,1]$. Here $\lambda$ is the proportion of investment going to the consumption goods sector. Since $H$ is a linear function of $\lambda$, maximizing $H$ with respect to $\lambda$ yields

$$
\begin{array}{ll}
\lambda=1, & \text { when } \Psi_{1}>\Psi_{2}, \\
\lambda \in[0,1], & \text { when } \Psi_{1}=\Psi_{2}, \\
\lambda=0, & \text { when } \Psi_{1}<\Psi_{2}
\end{array}
$$

There are three control variables: $\lambda, x_{f}, L_{f}$. Maximizing $H$ with respect to the last two variables and defining $\Psi=\max \left(\Psi_{1}, \Psi_{2}\right)$ we get:

$$
\begin{aligned}
U^{\prime}(C) / \Psi & =\frac{\delta M}{\delta L_{m}} / \frac{\delta F}{\delta L_{f}}, \\
U^{\prime}(C) / \Psi & =P .
\end{aligned}
$$

We can interpret $\Psi$ as the imputed price of investment goods in terms of utility. The transversality conditions require that $\Psi_{1}(0)$ and $\Psi_{2}(0)$ should be minimum values consistent with $\Psi_{1}(t)>0$ and $\Psi_{2}(t)>0$ for all $t \geqslant 0$. Along the optimal path $I>0$. If $I=0$, instead of Eq. (11) we get $U^{\prime}(C) \geqslant P \cdot \Psi$ with $C=F+M / P$. Since in this case the economy repeats itself over time, it can be shown that $\Psi_{1}(t)<0$ for some finite $t$. This violates the condition for optimality. The transversality conditions imply that $\Psi(t)$ assumes the minimum value among all possible choices of $\Psi(t)$ satisfying the necessary conditions. It can be shown that, for a higher value of $\Psi(0), \Psi^{\prime}(t)$ is higher at each point of time implying

Originally publlished in Journal of Economic Theory (1974), 7, 115-121 
a lower $C(t)$ for all $t \geqslant 0$. From Eqs. (10) and (11) we get the following:

$$
P=\frac{\delta M}{\delta L_{m}} / \frac{\delta F}{\delta L_{f}} .
$$

Equation (12) implies that the planners select a point on the transformation frontier such that the slope of the frontier at that point equals $P$. Equation (11) tells us how $\Psi$ determines the values of $C$ and $I$. The $\Psi_{i}$ 's determine the allocation of investment according to Eq. (9). Along the optimal path $\Psi_{1}(t)$ and $\Psi_{2}(t)$ must satisfy the following conditions:

$$
\begin{aligned}
& \dot{\Psi}_{1}=-U^{\prime}(C) \cdot \frac{\delta F}{\delta K_{f}}, \\
& \dot{\Psi}_{2}=-\Psi \cdot \frac{\delta M}{\delta K_{m}} .
\end{aligned}
$$

Equations (9) through (14) describe the necessary conditions which an optimal path must satisfy. Since along the optimal path the Hamiltonian assumes a constant value, say $\bar{H}$, Eqs. (6), (8), (9), and (11) yield

$$
I=P \cdot \frac{\bar{H}-U(C)}{U^{\prime}(C)},
$$

which determines the relation between $C$ and $I$ at each point of time.

\section{The Optimal Path}

In this section we shall prove a theorem regarding non-optimality of the myopic decision rule. Before doing so, we should explain a few things. Given our assumption of a fixed terms of trade, the locus of the points

$$
\frac{\delta M}{\delta K_{m}}=P \cdot \frac{\delta F}{\delta K_{f}}
$$

in the $K_{f} K_{m}$ plane is a straight line with negative slope. This critical path will be called Rybczynski Locus. A detailed discussion on Rybczynski Locus and the proof of the following proposition can be found in [3].

Propositron. If the consumption sector is relatively capital intensive, $\delta M / \delta K_{m}>\boldsymbol{P} \cdot \delta F / \delta K_{f}$ for any point below the Rybczynski Locus. On the other hand, $\delta M / \delta K_{m}<P \cdot \delta F / \delta K_{f}$ for any point above it. If the manufacturing sector is capital intensive, the result is reversed.

Originally publlished in Journal of Economic Theory (1974), 7, 115-121 
THEOREM 1. An optimal path which crosses the Rybczynski Locus camot be generated by the myopic decision rule.

Proof. Without any loss of generality, assume the consumption sector to be relatively capital intensive. Suppose there exists an optimal path which crosses the Rybczynski Locus and is consistent with myopic behavior. In other words, along this path, $\left(\Psi_{1}-\Psi_{2}\right)>0$ when

$$
\left(P \cdot \frac{\delta F}{\delta K_{f}}-\frac{\delta M}{\delta K_{m}}\right)>0
$$

and vice versa. Let $T$ denote the time when the path intersects the Rybczynski Locus with $\Psi_{1}(T)=\Psi_{2}(T)$. We shall show that such a path cannot be optimal.

Since $\Psi_{1}(t)$ and $\Psi_{2}(t)$ are continuous functions of $t$ (see Theorem 3 , p. 48 , of [5]),

$$
\lim _{\tau \rightarrow 0}\left[\Psi_{j}(T+\tau)-\Psi_{j}(T-\tau)\right]=0 ; \quad j=1,2 ; \quad \tau>0
$$

Let $\eta(t)=\Psi_{1}(t)-\Psi_{2}(t)$. Initially $\eta(t)<0$ and for $t>T, \eta(t)>0$.

$$
\frac{d \eta}{d t}=-U^{\prime}(C) \cdot \frac{\delta F}{\delta K_{f}}+\Psi \cdot \frac{\delta M}{\delta K_{m}}=\Psi \cdot\left(\frac{\delta M}{\delta K_{m}}-P \cdot \frac{\delta F}{\delta K_{f}}\right) .
$$

Consider $T-\tau$ for some $\tau>0$. Since at $T-\tau, \delta M / \delta K_{m}>P \cdot \delta F / \delta K_{f}$,

$$
d \eta / d t>0 \quad \text { at } \quad T-\tau
$$

Similarly,

$$
d \eta / d t<0 \quad \text { at } \quad T+\tau
$$

We also know that

$$
\begin{array}{ll}
\eta(t)<0, & \text { for } \quad 0 \leqslant t<T, \\
\eta(t)=0, & \text { for } t=T, \\
\eta(t)>0, & \text { for } \quad t>T .
\end{array}
$$

Conditions (17) through (19) imply that $\eta(t)$ must be discontinuous at $T$. Therefore either $\Psi_{1}(t)$ or $\Psi_{2}(t)$ or both are discontinuous at $T$. This contradicts Eq. (16). Hence such a path cannot be optimal.

When the consumption goods sector is capital intensive, it can be shown that asymptotically $\Psi=\Psi_{1}>\Psi_{2}, \lambda=1$. Otherwise $\Psi_{1}(t)<0$ for some finite $t$. Again, any optimal path which starts below the

Originally publlished in Journal of Economic Theory (1974), 7, 115-121 
Rybczynski Locus must cross the Locus. Otherwise, $\Psi_{2}(t)<0$ for some finite $t$. Asymptotically $\eta(t) \geqslant 0$, which implies

$$
\Psi_{2}(t) \leqslant \Psi_{1}(t)+\int_{t}^{\infty} \Psi(\tau)\left(\frac{\delta M}{\delta K_{m}}-P \cdot \frac{\delta F}{\delta K_{f}}\right) \cdot d \tau .
$$

It is easy to see that $\Psi_{1}(t)>\Psi_{2}(t)$, everywhere on or above the Rybczynski Locus. Suppose at $t$, the economy is somewhere on or above the Rybczynski Locus. Then, for all $\tau>t$, the expression under the sign of integration in Eq. (20) must be negative. Therefore by Eq. (20), $\Psi_{1}(t)>\Psi_{2}(t)$. This result implies that $\Psi_{1}(t)$ can equal $\Psi_{2}(i)$ only inside the Rybczynski Locus. In other words, the optimal switching locus must lie inside the Rybczynski Locus.

The situation is illustrated in Figure 1, which shows that myopic

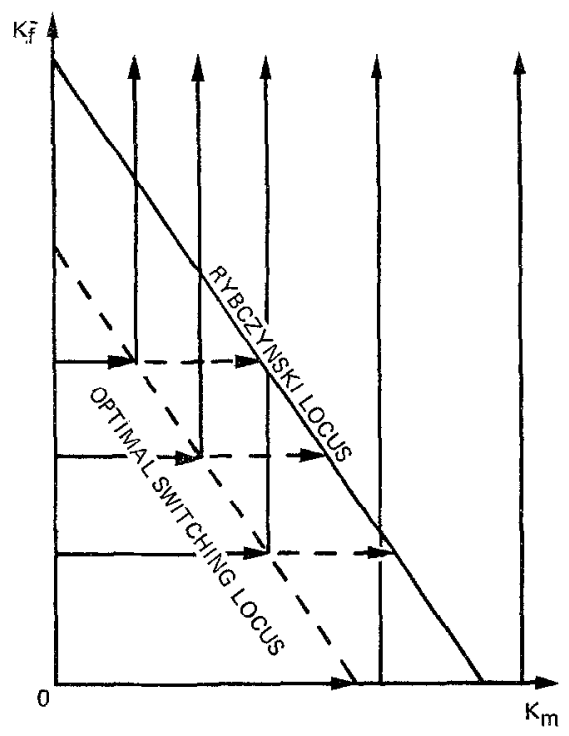

FIG. 1. The optimal paths.

decision under static price expectations is optimal everywhere except in the band between the optimal switching locus and the myopic switching (Rybczynski) locus.

Throughout this discussion we assumed that the supply of workers is fixed. If the number of workers is assumed to grow over time, a Rybczynski Locus can be drawn like the one in Figure 1, with $K_{j}$ 's being deflated by the total number of workers. Even in this case Theorem 1 holds true. However, when the number of workers is growing exponentially, it is

Originally publlished in Journal of Economic Theory (1974), 7, 115-121 
possible for the economy to run allong the Rybczynski Locus and approach a limit point (see [3, pp. 57-68]). This implies that an optimal path, after reaching the Rybczynski Locus, may lie on it forever. In this case, an optimal path need not cross the Rybczynski Locus, and the conditions for optimality may be consistent with the myopic decision rule.

\section{REFERENCES}

1. K. I. Arrow, Optimal Capital Policy, the Cost of Capital, and Myopic Decision Rules, Tech. Rept. No. 131, Institute for Mathematical Studies in Social Sciences, Stanford University, 1965.

2. K. J. ARrow, Optimal capital policy with irreversible investment, "Value, Capital, and Growth" (J. N. Wolfe, ed.), Aldine, Chicago, 1968, pp. 1-19.

3. T, K. Brswas, Nonshiftable capital and imported inputs in economic growth and International Trade, Ph.D. Dissertation, University of Rochester, June, 1972.

4. M. BRuno, Optimal capital accumulation in discrete capital models, "Essays on the Theory of Optimal Economic Growth" (K. Shell, ed.), M.I.T. Press, Cambridge, Mass. 1967, pp. 181-217.

5. L. S. PONTRYaGin et al., "The Mathematical Theory of Optimal Processes," Macmillan, New York, 1964.

6. K. Shel AND J. E. STIGLITZ, The allocation of investment in a dynamic economy, Quart. J. Econ. 81 (1967), 592-609.

Originally publlished in Journal of Economic Theory (1974), 7, 115-121 